\title{
DNA damage as a molecular link in the pathogenesis of COPD in smokers
}

\author{
Kazutetsu Aoshiba*,\#, Fang Zhou*, Takao Tsuji ${ }^{\#}$ and Atsushi Nagai ${ }^{\#}$
}

ABSTRACT: In this study, we investigated whether DNA double-strand breaks (DSBs) contribute to the pathogenesis of chronic obstructive pulmonary disease (COPD).

We immunofluorescence-stained lung tissue samples obtained from COPD patients, asymptomatic smokers and nonsmokers for markers of DSBs.

The numbers of DSB foci (phosphorylated histone 2AX $(\gamma \mathrm{H} 2 \mathrm{AX})$, phosphorylated ATM (ataxia telangiectasia mutated) substrate and phosphorylated p53-binding protein-1 foci) per cell in alveolar type I and II cells and endothelial cells were higher in the COPD patients than in the asymptomatic smokers and nonsmokers. The lung tissue in which type II cells contained higher numbers of $\gamma \mathrm{H} 2 \mathrm{AX}$ foci per cell had higher percentages of type II cells that expressed p16 ${ }^{\text {INK4a }}$ (p16), phosphorylated nuclear factor (NF)-кB and interleukin (IL)-6, and of alveolar wall cells that expressed active caspase-3. The type II cells that contained higher numbers of $\gamma \mathrm{H} 2 \mathrm{AX}$ foci per cell had higher rates of expression of $\mathbf{p 1 6}$, phosphorylated NF-kB, and IL-6. Half of the alveolar wall cells that expressed active-caspase-3 contained $\gamma \mathrm{H} 2 \mathrm{AX}$ foci. Type II cells that stained positive for 8-hydroxy-2-deoxyguanosine contained a higher number of $\gamma \mathrm{H} 2 \mathrm{AX}$ foci per cell than the type II cells that stained negative.

In conclusion, DSBs, at least in part caused by oxidative stress, appear to contribute to the pathogenesis of COPD by inducing apoptosis, cell senescence and pro-inflammatory responses.

KEYWORDS: Apoptosis, cell senescence, chronic obstructive pulmonary disease, DNA damage, interleukin-6, nuclear factor-кB

hronic obstructive pulmonary disease (COPD) is characterised by a persistent abnormal inflammatory response to noxious environmental stimuli, most commonly to cigarette smoke. Recent evidence indicates that the pathogenesis of COPD involves oxidative stress, apoptosis and cell senescence, as well as inflammation [1]. These multiple pathobiological processes in COPD are thought to be associated with the generation of interactive feedback loops that contribute to alveolar destruction, airway remodelling and ineffective tissue repair [1]. Although cigarette smoking directly causes inflammation, as well as oxidative stress, apoptosis and cell senescence, only a minority of smokers develop clinically significant COPD, and the inflammation and decline in pulmonary function continue even after smoking cessation [1]. This suggests the presence of specific mechanisms that are responsible for the susceptibility to damage by smoking, persistent inflammation and the chronic, progressive nature of the structural derangement in the lungs of COPD patients. We conducted the present study to test our hypothesis that DNA damage that alters the behaviour of pulmonary parenchymal cells underlies COPDspecific mechanisms.

Cigarette smoke is the most important environmental pollutant that causes DNA damage [2-4]. DNA double-strand breaks (DSBs) are among the most lethal forms of DNA damage caused by smoking [5], and if left unrepaired they can lead to apoptosis, cell senescence, pro-inflammatory responses and oncogenesis [6-8]. When a DSB is induced, the histone $2 \mathrm{~A}(\mathrm{H} 2 \mathrm{~A}) \mathrm{X}$ becomes rapidly phosphorylated at serine 139 by ATM (ataxia telangiectasia mutated)/ATR (ataxia telangiectasia and Rad3-related) and DNA-dependent protein kinase. This modified form, called $\gamma \mathrm{H} 2 \mathrm{AX}$, is easily identified by staining with antibodies and serves as a reliable, sensitive indicator of DSBs [7-10]. Since each DSB corresponds to one $\gamma \mathrm{H} 2 \mathrm{AX}$ focus, immunofluorescence-based assays that allow visualisation of discrete nuclear $\gamma \mathrm{H} 2 \mathrm{AX}$ foci are $\geqslant 100$ times more sensitive than
AFFILIATIONS

*Pulmonary Division, Graduate School of Medical Science, and ${ }^{\#}$ First Dept of Medicine, Tokyo Women's Medical University, Tokyo, Japan.

CORRESPONDENCE

K. Aoshiba

First Dept of Medicine

Tokyo Women's Medical University

8-1 Kawada-cho

Shinjuku-ku

Tokyo 162-8666

Japan

E-mail: kaoshiba@chi.twmu.ac.jp

\section{Received:}

March 222011

Accepted after revision:

Aug 292011

First published online:

Jan 202012 
other methods of detecting DSBs, such as pulsed-field gel electrophoresis and the alkaline comet assay [7-10]. $\gamma \mathrm{H} 2 \mathrm{AX}$ then recruits DNA repair proteins, including p53-binding protein (53BP)1, to the sites of DSBs and initiates DNA damage signal transduction that determines whether the DNA will be repaired; if repair is impossible, apoptosis, senescence or a pro-inflammatory response will be activated $[6,11,12]$. Thus, the persistence of $\gamma \mathrm{H} 2 \mathrm{AX}$ foci is regarded as indicating that some of the DNA damage remains unrepaired.

In the present study, we performed immunofluorescence staining for $\gamma \mathrm{H} 2 \mathrm{AX}$ foci, a marker of the presence of DSBs, to determine the intensity of DSB damage in the lungs of COPD patients. The results showed the presence of significantly more $\gamma \mathrm{H} 2 \mathrm{AX}$ foci in the alveolar type I and II cells and endothelial cells of the lungs of COPD patients than in the lungs of nonCOPD smokers and nonsmokers, and that the higher numbers of $\gamma \mathrm{H} 2 \mathrm{AX}$ foci were associated with apoptosis, cell senescence, pro-inflammatory phenotypic changes and DNA oxidation. These results suggest that DNA damage that is, at least in part, caused by oxidative stress contributes to the molecular pathogenesis of COPD by inducing apoptosis, cell senescence and pro-inflammatory responses.

\section{METHODS}

\section{Human lung tissue samples}

The protocol of the study conformed to the Declaration of Helsinki and approval was obtained from the Tokyo Women's Medical University Institutional Review Board, Tokyo, Japan (grant number 1783). Lung tissue blocks were obtained from COPD patients who were smokers (COPD smokers; $n=14$ ) during lung volume reduction surgery $(n=13)$ or pulmonary resection for localised lung cancer $(n=1)$, smokers who did not have COPD (non-COPD smokers; $n=10$ ), and nonsmokers $(n=10)$ during pulmonary resection for localised lung cancer. Each tissue block was fixed in $10 \%$ formalin, embedded in paraffin and cut into sections $3 \mu \mathrm{m}$ thick. All of the COPD and non-COPD smokers had stopped smoking $\geqslant 3$ months before the surgery. The patients' clinical information is shown in table 1 .

\section{Immunofluorescence staining}

Deparaffinised tissue sections were double or triple immunofluorescence stained using primary antibodies against $\gamma \mathrm{H} 2 \mathrm{AX}$, 53BP1, phosphorylated ATM/ATR substrate, p16 ${ }^{\text {INK4a }}$ (p16), cleaved (active) caspase-3, phosphorylated nuclear factor (NF)$\kappa \mathrm{B}$, interleukin (IL)-6, 8-hydroxy-2-deoxyguanosine (8-OHdG), surfactant protein (SP)-C, aquaporin (AQP)-5 and CD31 (see online supplementary material for details).

We examined $\geqslant 40$ randomly selected microscopic fields on each slide with an Olympus BX60 epifluorescence microscope (Olympus Optical Co. Ltd, Tokyo, Japan) equipped with a $100 \times$ objective lens, and visually counted the numbers of $\gamma \mathrm{H} 2 \mathrm{AX}$ foci, phosphorylated 53BP1 foci and phosphorylated ATM substrate foci per cell in SP-C-positive cells, the number of $\gamma \mathrm{H} 2 \mathrm{AX}$ foci per cell in AQP5-positive cells, and the number of $\gamma \mathrm{H} 2 \mathrm{AX}$ foci per cell in CD31-positive cells. We also counted the number of $\gamma \mathrm{H} 2 \mathrm{AX}$ foci per cell in SP-C-positive cells according to whether they expressed $\mathrm{p} 16$, phosphorylated NF$\kappa \mathrm{B}, \mathrm{IL}-6$ or $8-\mathrm{OHdG}$, and the number of $\gamma \mathrm{H} 2 \mathrm{AX}$ foci per cell in alveolar wall cells according to whether they expressed active caspase-3.

\section{Cell culture and irradiation}

Normal human lung microvascular endothelial cells were irradiated with a 10-Gy X-ray dose (see online supplementary material for details).

\section{Guinea pig exposure to cigarette smoke}

Hartley-strain guinea pigs were exposed to air or the smoke of 10 cigarettes 5 days a week for 10 weeks (see online supplementary material for details).

\section{Statistical analysis}

Statistical analyses were performed using Excel X (Microsoft Corp., Redmond, WA, USA) with the Statcel 3 add-in (OMS, Tokyo, Japan). Clinical, cell culture and animal experiment data are presented as mean \pm SEM, and human histological data are presented as medians. Differences in clinical data were analysed by ANOVA and if the results were significant, the Tukey-Kramer test was used as a multiple comparison post hoc test. Differences in human histological data were analysed by the Kruskall-Wallis test and if the results were significant, the Steel-Dwass multiple comparison test was used. Differences in cell culture data and animal experiment data were analysed by unpaired t-tests. Correlations were analysed by the Spearman rank correlation test. A p-value of $<0.05$ was considered statistically significant.

\section{RESULTS}

\section{Increased level of DSBs in the lungs of COPD patients}

A single $\gamma \mathrm{H} 2 \mathrm{AX}$ focus reflects hundreds to thousands of $\gamma \mathrm{H} 2 \mathrm{AX}$ protein molecules concentrated around one DSB [9]. As shown in figure 1a, immunofluorescence staining for $\gamma \mathrm{H} 2 \mathrm{AX}$ showed that the nuclei of many of the alveolar wall cells in the lungs of the COPD patients contained multiple $\gamma \mathrm{H} 2 \mathrm{AX}$ foci. No signal was visible when the anti- $\gamma \mathrm{H} 2 \mathrm{AX}$ antibody was omitted or the antibody was pre-incubated with

\begin{tabular}{|c|c|c|c|}
\hline & COPD patients & Smokers & Nonsmokers \\
\hline Subjects & 14 & 10 & 10 \\
\hline Males/females & $14 / 0$ & $10 / 0$ & $3 / 7$ \\
\hline Age yrs & $63.2 \pm 2.4$ & $61.6 \pm 4.2$ & $66.3 \pm 3.3$ \\
\hline Smoking pack-yrs & $74.4 \pm 13.2$ & $47.0 \pm 7.2$ & $0 \pm 0$ \\
\hline FEV1 L & $0.90 \pm 0.11^{* *}$ & $2.37 \pm 0.20$ & $2.05 \pm 0.11$ \\
\hline 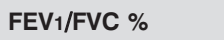 & $33.4 \pm 3.3^{\star \star}$ & $75.7 \pm 2.2$ & $75.3 \pm 1.8$ \\
\hline FEV $1 \%$ pred & $33.7 \pm 3.8^{* *}$ & $94.6 \pm 4.0$ & $97.2 \pm 5.2$ \\
\hline \multicolumn{4}{|l|}{ COPD severity } \\
\hline GOLD stage ॥ & 2 & & \\
\hline GOLD stage III & 4 & & \\
\hline GOLD stage IV & 8 & & \\
\hline
\end{tabular}

Data are presented as $\mathrm{n}$ or mean \pm SEM. All chronic obstructive pulmonary disease (COPD) patients and smokers were ex-smokers. FEV1: forced expiratory volume in $1 \mathrm{~s}$; FVC: forced vital capacity; \% pred: \% predicted; GOLD: Global Initiative for Chronic Obstructive Lung Disease. ${ }^{* *}: \mathrm{p}<0.01$ compared with asymptomatic smokers and nonsmokers. 

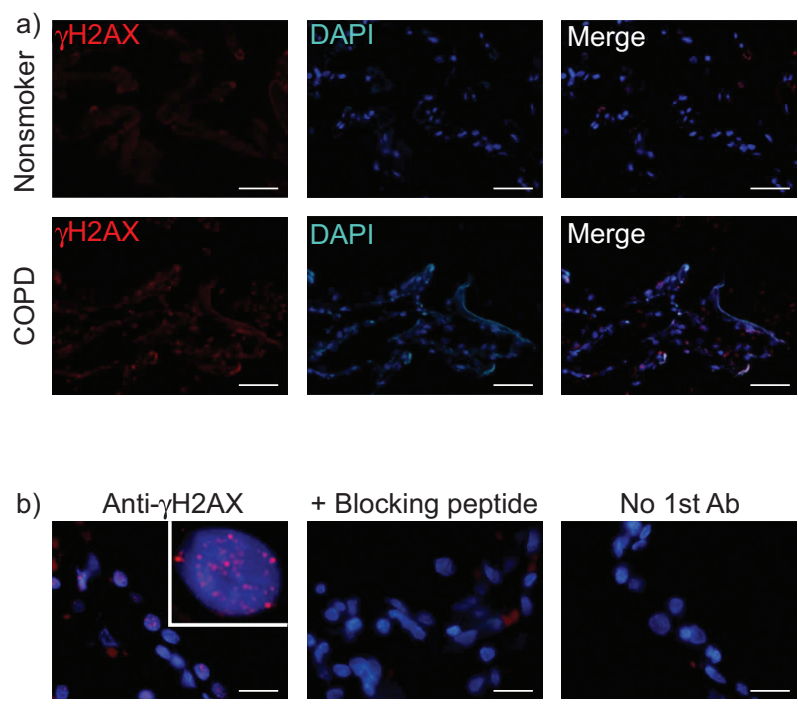

FIGURE 1. a) Representative images of anti-phosphorylated histone $2 A X$ $(\gamma \mathrm{H} 2 \mathrm{AX})$ immunofluorescence-stained sections of lung tissue obtained from asymptomatic nonsmokers and chronic obstructive pulmonary disease (COPD) patients. Nuclear DNA was counterstained with 4,6-diamidino-2-phenylindole (DAPI). Scale bars $=50 \mu \mathrm{m}$. b) Specificity of $\gamma \mathrm{H} 2 \mathrm{AX}$ immunofluorescence staining. Discrete $\gamma \mathrm{H} 2 \mathrm{AX}$ foci in the nuclei of alveolar wall cells in the lungs of COPD patients were no longer visible when the antibody was pre-incubated with a blocking peptide before application of the secondary antibody or the anti- $\gamma \mathrm{H} 2 \mathrm{AX}$ antibody was omitted. Ab: antibody. Scale bars $=20 \mu \mathrm{m}$.

a blocking peptide, thereby verifying the specificity of the $\gamma \mathrm{H} 2 \mathrm{AX}$ signal (fig. 1b). As shown in figure $2 \mathrm{~b}$, the $\gamma \mathrm{H} 2 \mathrm{AX}$ foci were co-localised with other DSB markers, including phosphorylated 53BP1 and phosphorylated ATM/ATR substrates $[13,14]$, thereby corroborating the validity of using $\gamma \mathrm{H} 2 \mathrm{AX}$ as a biomarker to measure DSB levels.

Quantitative analyses showed greater numbers of $\gamma \mathrm{H} 2 \mathrm{AX}$ foci per cell in AQP5-positive type I cells, SP-C-positive type II cells and CD31-positive endothelial cells in the COPD patients than in the non-COPD smokers and the nonsmokers (figs $2 \mathrm{a}$ and $3 \mathrm{a}-\mathrm{c})$. Similarly, the numbers of phosphorylated 53BP1 foci and phosphorylated ATM/ATR substrate foci per cell in type II cells were significantly higher in the COPD smokers than in the non-COPD smokers and nonsmokers (figs $2 \mathrm{a}$, and $3 \mathrm{a}$ and e). However, the type I and II cells and endothelial cells of the non-COPD smokers and nonsmokers were found to contain similar numbers of $\gamma \mathrm{H} 2 \mathrm{AX}$ foci, phosphorylated 53BP1 foci and phosphorylated ATM/ATR substrate foci per cell (fig. 3). The numbers of $\gamma \mathrm{H} 2 \mathrm{AX}$ foci per cell in type II cells were closely correlated with the numbers of phosphorylated 53BP1 foci per cell $(r=0.83)$ and phosphorylated ATM/ATR substrate foci per cell $(r=0.78)$ in type II cells (fig. S1). These results suggest the presence of more severe DNA damage in the alveolar type I and II cells and endothelial cells of the COPD patients than in those of the non-COPD smokers and nonsmokers.

\section{Tissue-level analyses of the relationship between DSBs and apoptosis, cell senescence, inflammation and oxidative stress}

Consistent with the results of previous studies [15-21], we found that the lungs of the COPD patients contained higher percentages of alveolar wall cells that expressed active caspase-3 (a marker of apoptosis) (fig. 4a), type II cells that expressed p16 (a marker of senescence) (fig. 4b), type II cells

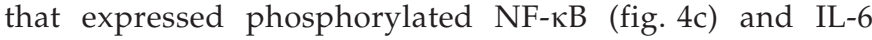
(fig. 4d) (markers of pro-inflammatory phenotypic changes), and type II cells that expressed 8-OHdG (a marker of oxidative stress) (fig. 4e) than the lungs of the non-COPD smokers and nonsmokers. Since DSB damage is a strong inducer of apoptosis, cell senescence and pro-inflammatory cytokine production $[6,11,12]$, we investigated whether the presence of $\gamma \mathrm{H} 2 \mathrm{AX}$, a marker of DSBs, was associated with apoptosis, cell senescence and pro-inflammatory phenotypic changes in type II cells. When all of the subjects were included in a tissue-level correlation analysis, the lung tissue with type II cells containing higher numbers of $\gamma \mathrm{H} 2 \mathrm{AX}$ foci contained higher percentages of alveolar wall cells that expressed active caspase-3 (fig. 5a), and higher percentages of type II cells that expressed p16 (fig. 5b), type II cells that expressed phosphorylated NF- $\mathrm{BB}$ (fig. $5 \mathrm{c}$ ) and type II cells that expressed IL-6 (fig. 5d). These results indicate that more severe DNA damage in lung tissue was associated with greater numbers of type II cells undergoing apoptosis, senescence, pro-inflammatory phenotypic changes and DNA oxidation.

\section{Cellular level analyses of the relationship between DSBs and apoptosis, cell senescence, inflammation and oxidative stress}

Next, we conducted a cellular level analysis to investigate whether the DNA damage was directly related to apoptosis, cell senescence and pro-inflammatory phenotypic changes. Analysis of the type II cells of the COPD patients showed that the cells that contained higher numbers of $\gamma \mathrm{H} 2 \mathrm{AX}$ foci had higher rates of expression of p16 (fig. 6a), phosphorylated NF- $\kappa B$ (fig. 6b) and IL-6 (fig. 6c). Almost all of the type II cells that contained $\geqslant 21 \gamma \mathrm{H} 2 \mathrm{AX}$ foci expressed $\mathrm{p} 16$ and phosphorylated NF- $\kappa$ B. Moreover, about half of the alveolar wall cells that expressed active caspase- 3 contained $\gamma \mathrm{H} 2 \mathrm{AX}$ foci (focal staining pattern), which indicated that the DNA damage had triggered apoptosis, whereas the remainder exhibited a diffuse $\gamma \mathrm{H} 2 \mathrm{AX}$ staining pattern, which indicated that DNA fragmentation was occurring during apoptosis (fig. 6d) [22]. These findings suggest that DNA damage is directly linked to the induction of apoptosis, cell senescence and pro-inflammatory phenotypic changes in the lungs of COPD patients.

Since oxidative stress is among the major causes of DSBs [23], we attempted to determine whether there was a correlation between DNA oxidation and the presence of $\gamma \mathrm{H} 2 \mathrm{AX}$ foci. A tissue-level analysis of the lungs of all of the subjects as a whole showed that the lung tissue with type II cells containing higher numbers of $\gamma \mathrm{H} 2 \mathrm{AX}$ foci per cell had higher percentages of type II cells that expressed 8-OHdG (fig. 5e). Moreover, a cellular level analysis of the lungs of the COPD patients alone showed that the type II cells that expressed 8-OHdG contained significantly more $\gamma \mathrm{H} 2 \mathrm{AX}$ foci than the type II cells that did not express 8-OHdG (fig. 6e). These results suggest that oxidative stress is at least partly responsible for DNA DSBs in the parenchymal cells of the lungs of COPD patients. 

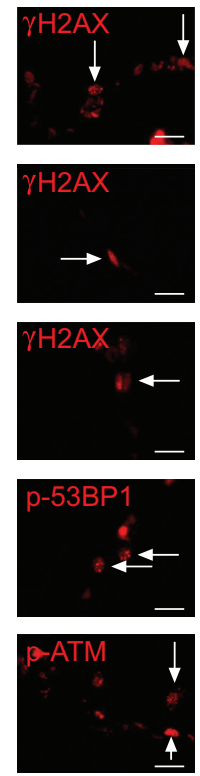

b)
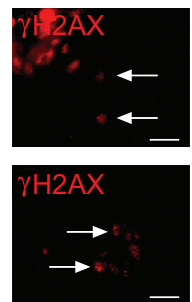
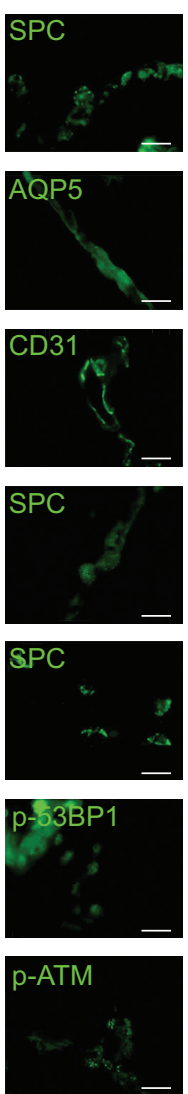
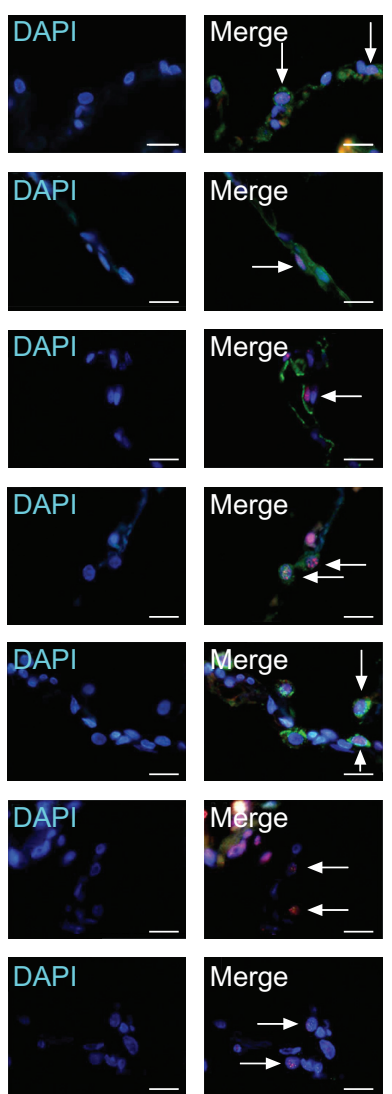
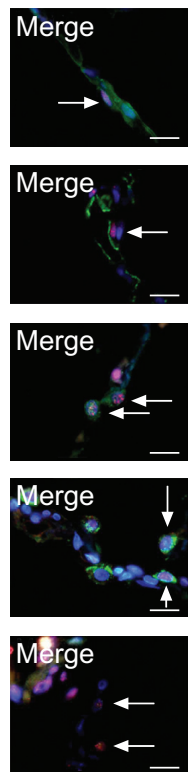

Merge

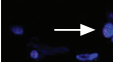

C)
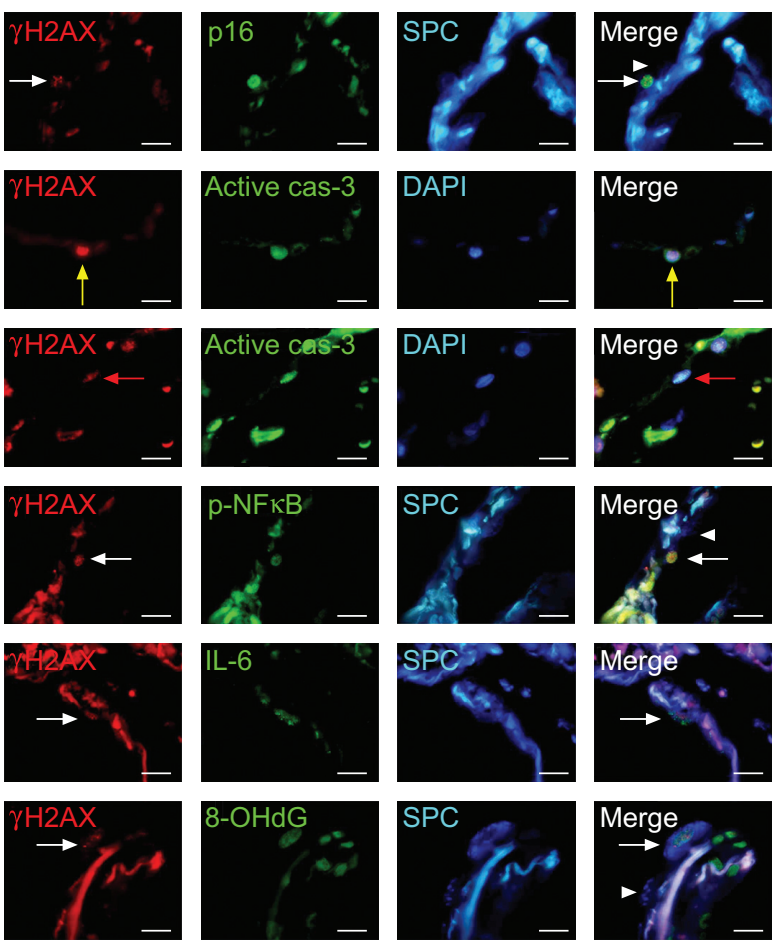

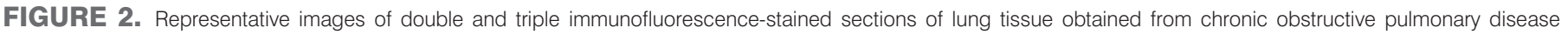

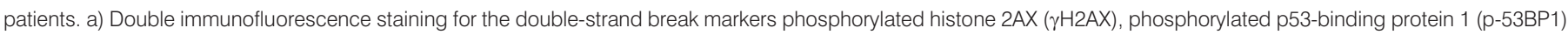

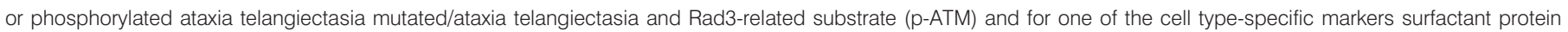

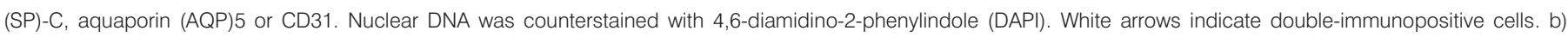

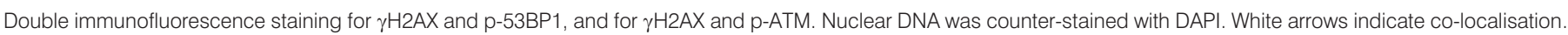

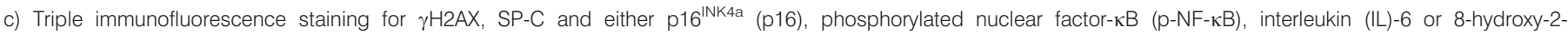

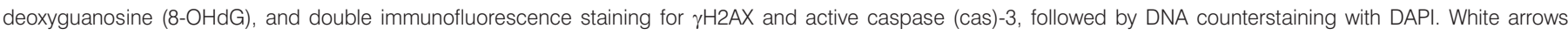

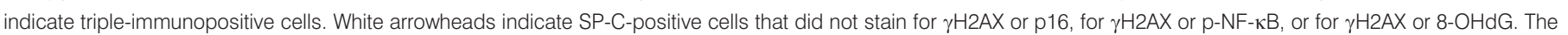
nuclei of active cas-3-positive cells stained diffusely (yellow arrows) or focally (red arrows) for $\gamma \mathrm{H} 2 \mathrm{AX}$. Scale bars $=20 \mu \mathrm{m}$.

\section{In vitro effects of DSBs on apoptosis, cell senescence and inflammation}

To confirm that DNA DSBs directly cause apoptosis, cell senescence and a pro-inflammatory response, we irradiated normal human lung microvascular endothelial cells with a single 10-Gy X-ray dose. This dose increased the numbers of $\gamma \mathrm{H} 2 \mathrm{AX}$ foci per cell three-fold and the numbers of phosphorylated 53BP1 foci per cells six-fold, thereby validating the induction of DSBs by X-irradiation (fig. S2). X-irradiation also increased the percentages of lung microvascular endothelial cells that stained positively for active caspase-3, p16 and phosphorylated NF-KB, and the amount of IL-6 secreted by lung microvascular endothelial cells (fig. S2). These results indicate that DNA DSBs directly caused apoptosis, cell senescence and a pro-inflammatory response in lung microvascular endothelial cells. We also treated the alveolar type II cell-like epithelial cell line A549 cells with bleomycin, which is known to induce both single-strand breaks and DSBs [24]. A549 cells express p21 but not p16 [25]. We found that bleomycin treatment also increased the numbers of $\gamma \mathrm{H} 2 \mathrm{AX}$ and phosphorylated 53BP1 foci per cell, the percentages of A549 cells that stained positively for active caspase-3, p21 and phosphorylated NF-KB, and the amount of IL-6 secreted by A549 cells (fig. S3).

\section{Increased DSBs in a guinea pig model of cigarette smoke- induced emphysema}

We also determined whether exposing guinea pigs to cigarette smoke causes DNA DSBs in the alveolar wall cells of their emphysematous lungs. Histological examination of samples of the lung tissue of the guinea pigs exposed to cigarette smoke for 10 weeks showed enlargement of the alveolar air spaces and the Lm value (mean airspace size) was $45 \%$ higher than in the nonsmoking control group $(\mathrm{p}<0.01)$ (fig. S4). The alveolar wall cells of the lungs of the cigarette smoke-exposed group contained twice as many $\gamma \mathrm{H} 2 \mathrm{AX}$ foci per cell as the alveolar wall cells of the lungs of the control group (mean \pm SEM $2.05 \pm 0.26$ versus $1.06 \pm 0.17 ; \mathrm{p}<0.01$ ) (fig. S4). These results indicate that DSBs occurred in the alveolar wall cells of emphysematous lungs of guinea pigs exposed to cigarette smoke. 

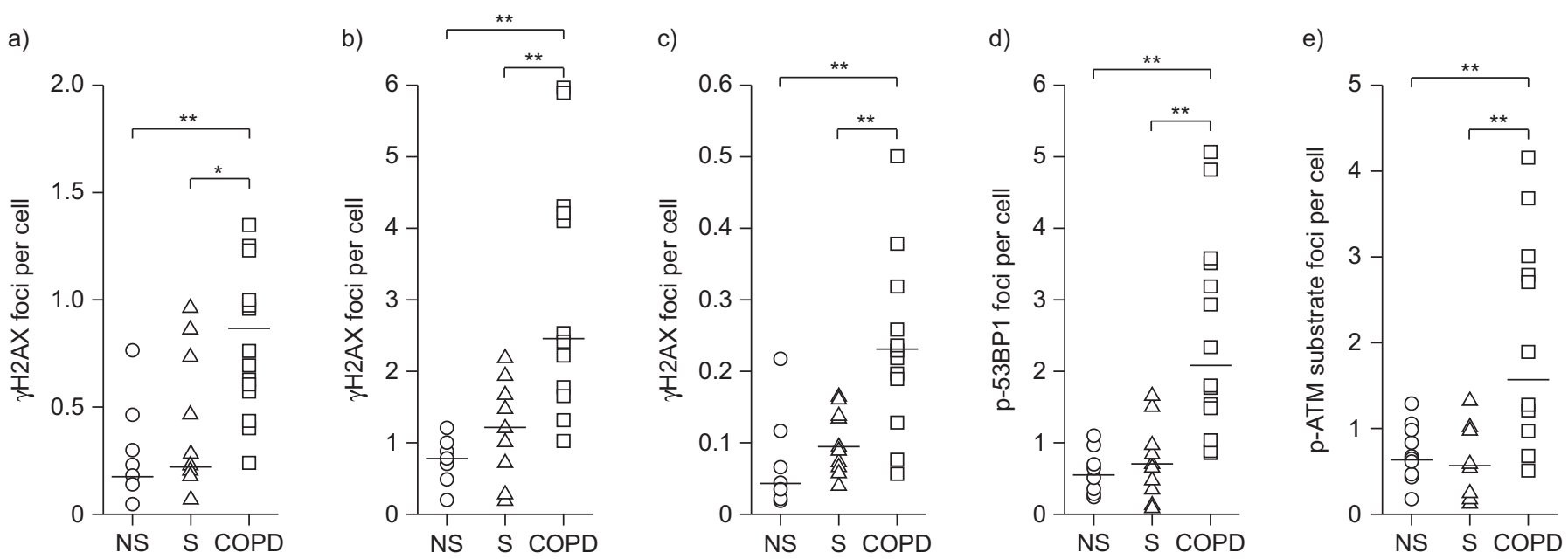

FIGURE 3. Quantitative analyses of DNA double-strand breaks in alveolar wall cells of the lungs of chronic obstructive pulmonary disease (COPD) patients, and asymptomatic smokers (S) and nonsmokers (NS). a) Phosphorylated histone 2AX ( $\gamma \mathrm{H} 2 \mathrm{AX}$ ) foci per cell in aquaporin 5-positive type I cells, b) surfactant protein (SP)-Cpositive type II cells and c) CD31-positive endothelial cells, and the numbers of d) phosphorylated p53-binding protein 1 ( $p$-53BP1) foci and e) phosphorylated ataxia telangiectasia mutated ( $p$-ATM) substrate foci per cell in SP-C-positive type II cells. —: median. *: $p<0.05 .{ }^{* *}: p<0.01$.

\section{DISCUSSION}

Immunofluorescence-based assays of $\gamma \mathrm{H} 2 \mathrm{AX}$ provide a sensitive, efficient and reproducible method of measuring the number of DSBs [7-10]. Since persistence of $\gamma \mathrm{H} 2 \mathrm{AX}$ foci after the initial induction of DNA DSBs indicates that some of the damage remains unrepaired, the persistence of foci is widely used as a biomarker of DNA damage in various tissues [7-10]. In the present study, we found significantly increased numbers of $\gamma \mathrm{H} 2 \mathrm{AX}$ foci in the alveolar wall cells, including type I and II cells, and endothelial cells of COPD smokers than in nonCOPD smokers and nonsmokers. The presence of $\gamma \mathrm{H} 2 \mathrm{AX}$ foci in type II cells was associated with apoptosis, cell senescence, pro-inflammatory phenotypic changes and oxidative stress. The results of this study also showed that the $\gamma \mathrm{H} 2 \mathrm{AX}$ foci were co-localised with DNA damage repair proteins, i.e. phosphorylated ATM/ATR substrates and phosphorylated 53BP1, and that the numbers of $\gamma \mathrm{H} 2 \mathrm{AX}$ foci per cell were closely correlated with the numbers of phosphorylated 53BP1 foci and phosphorylated ATM/ATR substrate foci per cell, thereby demonstrating the validity of $\gamma \mathrm{H} 2 \mathrm{AX}$ number as a biomarker of DNA damage. Taken together, the results of the present study suggest that DNA damage, in particular DSBs caused at least in part by oxidative stress, contributes to the molecular pathogenesis of COPD by inducing apoptosis, cell senescence and pro-inflammatory responses.

The results of this study provide evidence that DSBs are a prominent feature of the alveolar wall cells, including type I and II cells and endothelial cells, of COPD patients. This evidence obtained in the human lung tissue study was supported by the results of our animal study in guinea pigs that showed significantly higher numbers of $\gamma \mathrm{H} 2 \mathrm{AX}$ foci in the
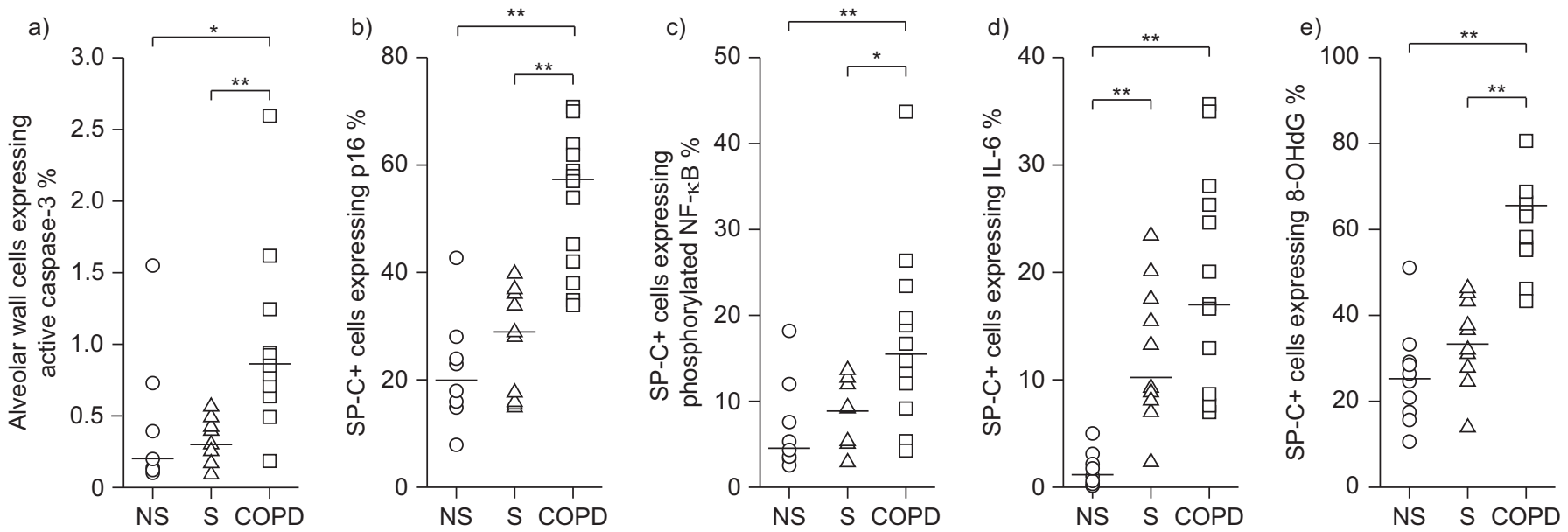

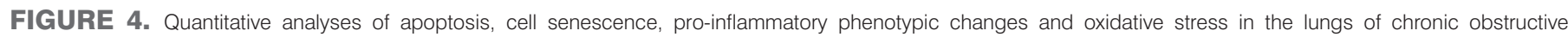

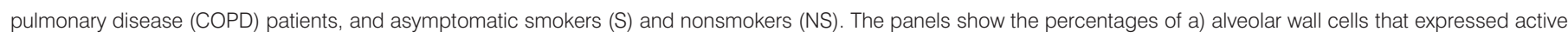

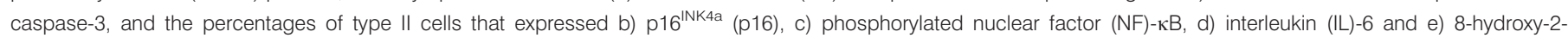
deoxyguanosine (8-OHdG). —: median values. SP: surfactant protein. *: $p<0.05$. **: $p<0.01$. 

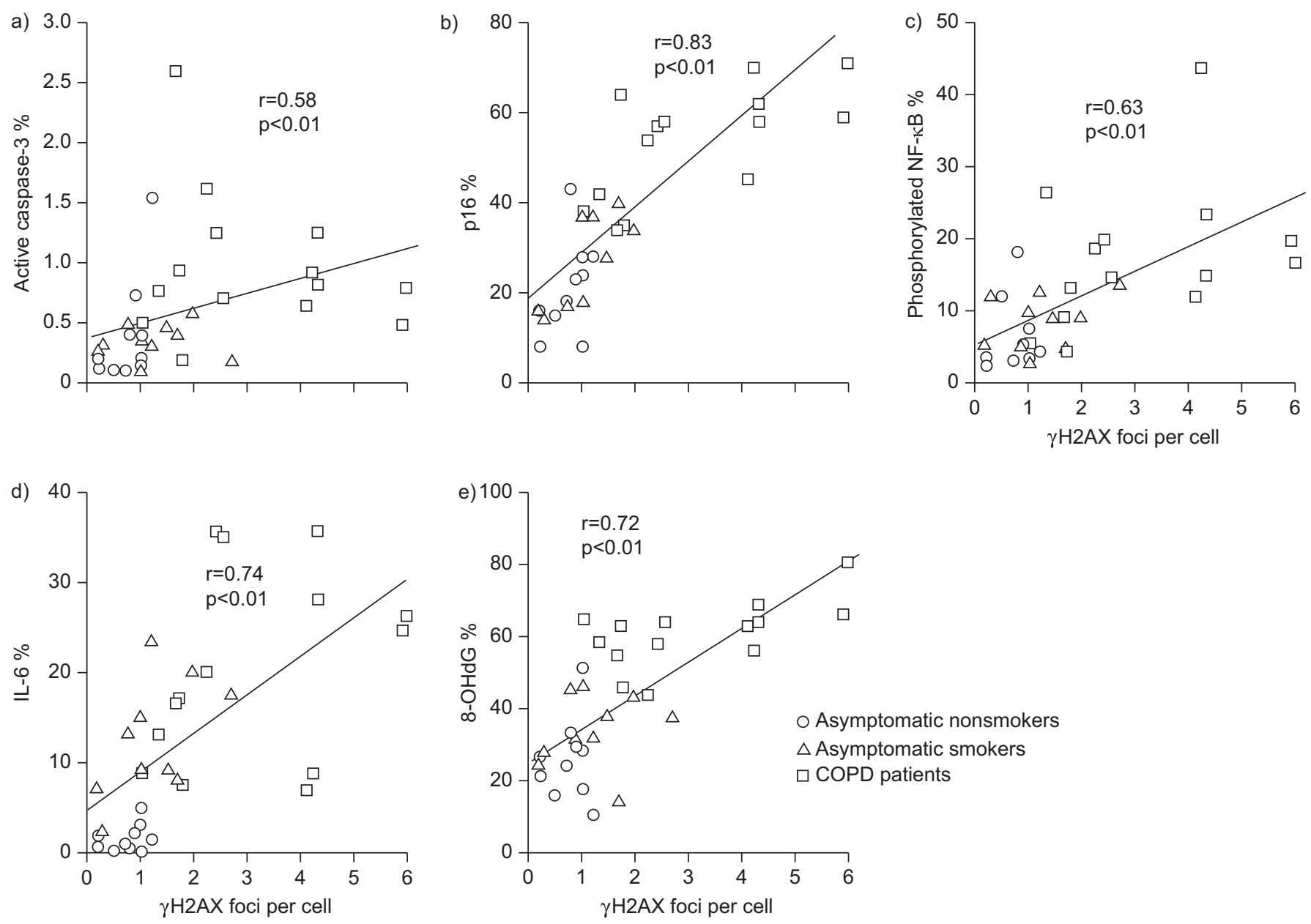

FIGURE 5. Tissue-level analyses of the relationships between the numbers of phosphorylated histone $2 \mathrm{AX}(\gamma \mathrm{H} 2 \mathrm{AX})$ foci per cell and the percentages of cells that stained positive for markers of apoptosis, cell senescence, pro-inflammatory phenotypic changes and oxidative stress in the entire group of subjects. The panels show positive correlations between the numbers of $\gamma \mathrm{H} 2 \mathrm{AX}$ foci per cell in type II cells and percentages of a) alveolar wall cells that expressed active caspase-3, and between the number of $\gamma \mathrm{H} 2 \mathrm{AX}$ foci per cell in type II cells and percentages of type II cells that expressed b) p16 $6^{\mathrm{INK} 4 a}$ (p16), c) phosphorylated nuclear factor (NF)-KB, d) interleukin (IL)-6 and e) 8 hydroxy-2-deoxyguanosine (8-OHdG). COPD: chronic obstructive pulmonary disease.

alveolar wall cells of the lungs of guinea pigs with cigarette smoke-induced emphysema than in the lungs of sham-exposed animals (fig. S4). Our findings are consistent with those of others [26-28], who detected DNA lesions in the form of microsatellite instability (MSI) and loss of heterozygosity $(\mathrm{LOH})$ in the sputum cells and bronchoalveolar fluid (BALF) cells obtained from COPD patients. Cigarette smoke contains many genotoxins, including benzo(a)pyrene, nitrosamines, aldehydes and oxidants, that induce various forms of DNA adducts [5]. The DNA damage accumulates at multiple genetic loci and sometimes induces DSBs, the most cytotoxic damage; these DSBs trigger the formation of $\gamma \mathrm{H} 2 \mathrm{AX}$ foci at the break site [29]. The results of the present study revealed significantly higher numbers of $\gamma \mathrm{H} 2 \mathrm{AX}$ foci per cell in the alveolar wall cells of COPD smokers, but not of non-COPD smokers, than in nonsmokers. This finding corroborates the findings of others [26-28], who detected MSI and LOH in sputum cells and BALF cells of COPD smokers but not of non-COPD smokers. Their findings, together with our own, strongly suggest that the DNA damage in the lungs of COPD smokers is amplified and/ or remains unrepaired, and this results in gradual accumulation of DNA damage in their lungs.

The current theory of the pathogenesis of COPD suggests that alveolar destruction is caused by interactions between several pathobiological processes, including inflammation, apoptosis, cell senescence and oxidative stress [1]. By carefully analysing correlations at both the tissue and cellular levels, we found that DNA damage is correlated with apoptosis, cell senescence and pro-inflammatory phenotypic changes in the lungs of COPD patients, thereby underscoring DNA damage as a molecular link between the pathobiological processes thought to be involved in the alveolar destruction in COPD.

Since this was an observational study of human lung tissue specimens, it was impossible to establish a causal connection between DNA damage and the development of apoptosis, cell senescence and pro-inflammatory phenotypic changes. Some of the DNA damage observed in this study may have been the result of apoptosis, cell senescence and inflammation rather than their cause. However, it is well established that DNA 


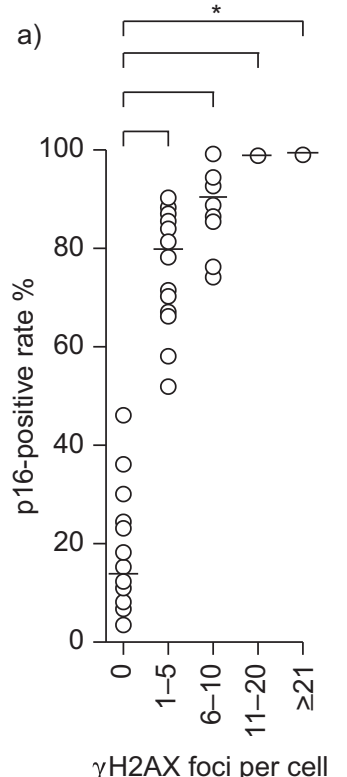

$\gamma \mathrm{H} 2 \mathrm{AX}$ foci per cell b)
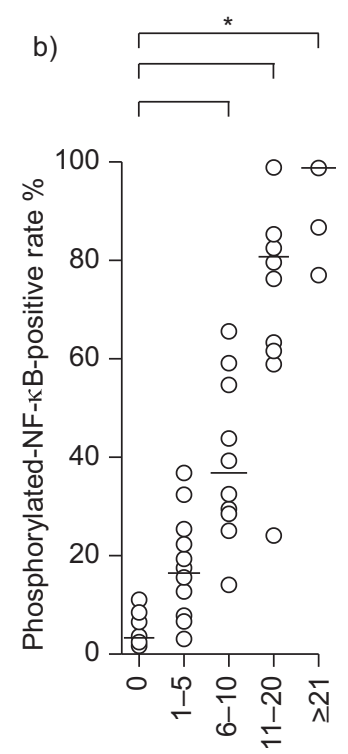

$\gamma \mathrm{H} 2 \mathrm{AX}$ foci per cell c)
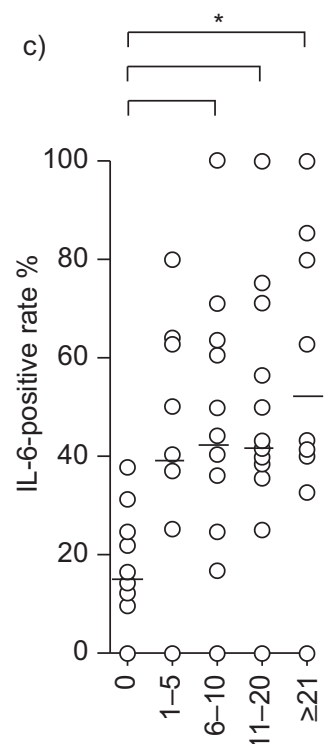

$\gamma \mathrm{H} 2 \mathrm{AX}$ foci per cell d)
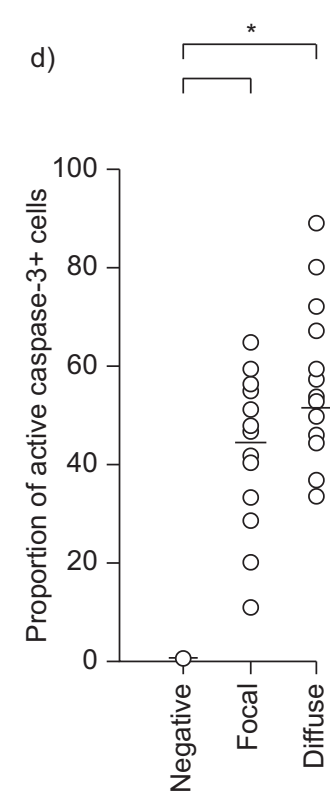

$\gamma \mathrm{H} 2 \mathrm{AX}$ staining pattern e)

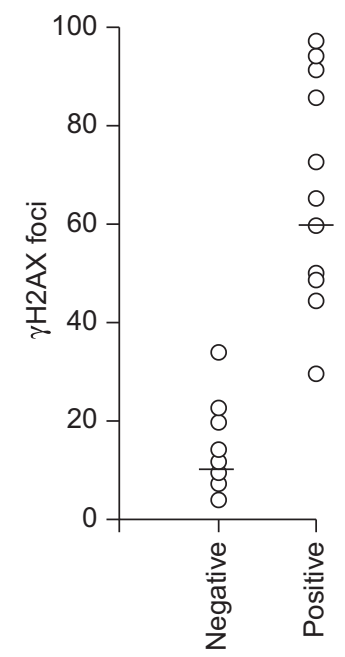

8-OHdG staining

FIGURE 6. Cellular level analyses of the relationships between the numbers of phosphorylated histone $2 \mathrm{AX}(\gamma \mathrm{H} 2 \mathrm{AX})$ foci per cell and the percentages of cells that stained positively for markers of apoptosis, cell senescence, pro-inflammatory phenotypic changes and oxidative stress in chronic obstructive pulmonary disease (COPD) patients. Percentages of type II cells that expressed a) $\left.\mathrm{p} 16^{\mathrm{INK} 4 \mathrm{a}}(\mathrm{p} 16), \mathrm{b}\right)$ phosphorylated nuclear factor (NF)- $\mathrm{KB}$ and c) interleukin (IL)-6 according to the numbers of $\gamma \mathrm{H} 2 \mathrm{AX}$ foci per cell. d) Proportion of active caspase-3-positive alveolar wall cells that exhibited no $\gamma \mathrm{H} 2 \mathrm{AX}$ staining, focal $\gamma \mathrm{H} 2 \mathrm{AX}$ staining and diffuse $\gamma \mathrm{H} 2 \mathrm{AX}$ staining (see text for explanation). e) Number of $\gamma \mathrm{H} 2 \mathrm{AX}$ foci per cell in type Il cells that expressed or did not express 8-hydroxy-2-deoxyguanosine (8-OHdG). Mean values obtained in an individual COPD patient are shown. - median. ${ }^{*}: p<0.05 .{ }^{*}: p<0.01$.

damage, in particular DSBs, is a strong inducer of apoptosis, cell senescence and pro-inflammatory responses in various types of cells and tissues [6-12]. In fact, many studies have shown that activation of an ATM $/ \gamma \mathrm{H} 2 \mathrm{AX}$-mediated signal transduction pathway in response to unrepaired DSBs causes apoptosis and cell senescence, thereby eliminating the DNAdamaged cells from the tissue and preventing their oncogenic transformation [6, 9]. Furthermore, a novel ATM $/ \gamma \mathrm{H} 2 \mathrm{AX}$ mediated pro-inflammatory signal transduction pathway in response to DSBs has recently been discovered that activates NF-KB and CCAAT/enhancer-binding protein- $\beta$ transcriptional

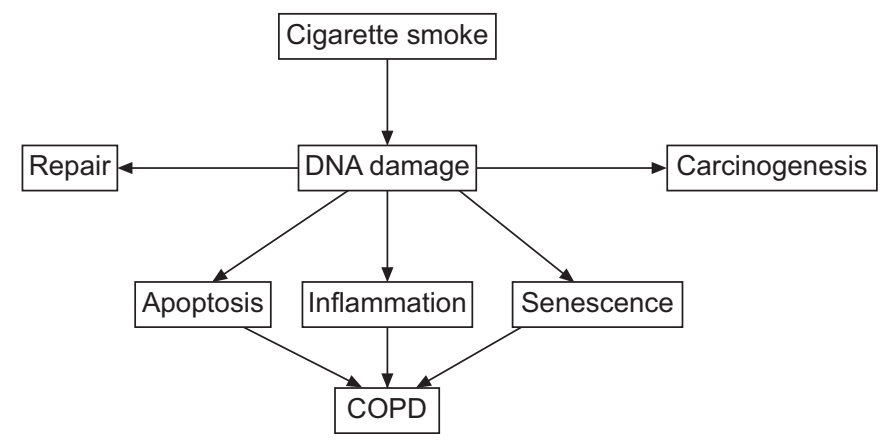

FIGURE 7. A DNA damage hypothesis for the pathogenetic mechanism of chronic obstructive pulmonary disease (COPD) in smokers. Cigarette smoke causes DNA damage, at least in part through oxidative stress. If left unrepaired, DNA damage induces apoptosis, cell senescence and a pro-inflammatory response of the alveolar wall cells, all of which contribute to the development of COPD. Unrepaired DNA damage also promotes carcinogenesis. activity and stimulates secretion of pro-inflammatory cytokines, including IL-6, which in turn acts in an autocrine feedback loop to reinforce the senescence growth arrest [11, 12]. In the present study, we showed that DSBs caused by X-irradiation of cultured lung microvascular endothelial cells induced pro-inflammatory responses, such as NF- $\mathrm{KB}$ phosphorylation and IL-6 production, as well as caspase- 3 activation and p16 expression, suggesting that DSBs are direct causes of pro-inflammatory responses, apoptosis and cell senescence (fig. S2). We also showed that treatment of A549 cells with bleomycin, which induced $\gamma \mathrm{H} 2 \mathrm{AX}$ focus formation, caused NF-kB phosphorylation and IL-6 production, as well as caspase-3 activation and p21 expression (fig. S3). We also recently found that persistent DNA damage in Clara cells induced by repeated injection of mice with naphthalene and bromo-2-deoxyuridine caused airway epithelial senescence accompanied by a p38 mitogen-activated protein kinase (MAPK)-dependent airway inflammatory response [30]. These lines of evidence, although not wholly conclusive, support the hypothesis that DNA damage plays a causative role in the apoptosis, cell senescence and pro-inflammatory responses observed in the lungs of COPD patients. However, future studies on animal models of COPD will be needed to show whether this view is correct.

Oxidative stress is among the major causes of DSBs [23]. In the present study, we demonstrated an association between the presence of $\gamma \mathrm{H} 2 \mathrm{AX}$ foci and the presence of 8-OHdG, suggesting that oxidative stress is responsible for the DSBs in the lungs of COPD patients. It has been proposed that oxidative stress contributes to apoptosis, cell senescence and inflammation in COPD $[1,31]$, and recent evidence suggests 
that oxidative stress induces inflammation via multiple mechanisms that involve oxidative activation of c-Jun activated kinase, p38-MAPK, NF- $\mathrm{B}$ and activator protein-1, and oxidative inhibition of histone deacetylase activity [31]. Our findings in this study show that oxidative DNA damage may also contribute to the mechanism of persistent inflammation in COPD.

The results of the present study showed that $\gamma \mathrm{H} 2 \mathrm{AX}$ focus formation was also associated with IL-6 production by type II cells. This finding corroborates recent evidence that "danger signals" from injured cells elicit an immune response [32], and that IL-6 is produced by DNA-damaged cells and is a major secreted factor in their pro-inflammatory phenotype [11, 12, 33]. It has been shown that IL-6 is involved in pulmonary and systemic inflammation in COPD [34] and that higher serum IL-6 levels are associated with lower forced expiratory volume in $1 \mathrm{~s}$ [35]. Overexpression of IL-6 in mice was found to result in the development of emphysema and airway fibrosis [36]. In view of these findings, we suggest that IL-6 may be a key mediator that links DNA damage to the inflammatory responses in COPD.

There were several limitations to this study. First, the COPD patients had a 1.6-fold greater smoking pack-yr history than the non-COPD smokers, although the difference between the two groups was not statistically significant (table 1$)(p=0.12$ by unpaired t-test). This possible mismatch regarding smoking intensity, however, is unlikely to have accounted for the higher numbers of $\gamma \mathrm{H} 2 \mathrm{AX}$ foci per cell in the COPD smokers, because no significant correlations were found between the smoking pack-yrs and the numbers of $\gamma \mathrm{H} 2 \mathrm{AX}$ foci per cell in type I cells $(r=0.16, p=0.58)$, type II cells $(r=0.001, p=0.99)$ or endothelial cells $(r=0.18, p=0.22)$. Secondly, it remains uncertain whether DNA damage is an early pathobiological event in COPD, because most of the COPD patients included in the present study had advanced COPD that received lung volume reduction surgery. A further study evaluating the number of $\gamma \mathrm{H} 2 \mathrm{AX}$ foci in all COPD stages, in subjects with different degrees of smoking exposure and in COPD patients treated with different medications would enable us to better elucidate the DNA damage in COPD. Thirdly, this study may have overestimated the level of DNA damage in the lungs of the non-COPD smokers and nonsmokers, because a comorbid cancer might have promoted the formation of $\gamma \mathrm{H} 2 \mathrm{AX}$ foci in the surrounding lung tissue through the bystander effect [37]. Finally, it remains unknown whether DNA damage in nonparenchymal cells, such as airway epithelial cells and pulmonary arterial endothelial cells, is also more severe in COPD patients.

Based on the results of the present study, we hypothesise that the apoptosis, cell senescence and inflammation, which are thought to represent the pathobiological processes of COPD [1], are at least partly attributable to DNA damage (fig. 7). The traditional theory of the pathogenesis of COPD suggests that activation of inflammation by inhaled cigarette smoke and other pollutants plays a central role in airway wall thickening, alveolar destruction, airspace enlargement and vascular remodelling [38]. Our hypothesis that DNA damage underlies the molecular mechanism of COPD seems to suggest answers to several important questions that the traditional theory does not address. The first question is, why does COPD take decades to develop? The answer based on our DNA damage hypothesis would be that the DNA damage caused by long-term smoking needs to accumulate over several decades before COPD develops, by analogy to the development of lung cancer. The second question is, why does inflammation persist after ceasing to smoke? The answer is that it probably persists because smoking-induced DNA damage persists long after smoking cessation, as is reported previously $[3,4]$. The third question is, why do corticosteroids have little impact on the inflammation in COPD? The answer may be that corticosteroids do not restore the DNA damage. Finally, why is it that some smokers develop COPD while others do not, and, why are COPD smokers more prone to develop lung cancer than non-COPD smokers? The answer to the last two questions would be that the greater susceptibility to DNA damage due to smoking may be genetically determined just as greater susceptibility to smoking-induced lung cancer, so that smokers who are more susceptible to DNA damage may be predisposed to both COPD and lung cancer. However, the results of the current study do not answer all these question; longitudinal studies will be needed that include a larger number of COPD patients with different stages of disease severity.

Two independent groups of investigators have recently proposed a somatic mutation hypothesis of COPD, which differs from our hypothesis in requiring a "somatic gene mutation" to explain enhanced inflammatory responses in COPD. In 2003, ANDERSON and BOZINOVSKI [39] proposed that acquired somatic mutations in the genes encoding p53, Ras, epidermal growth factor receptor and PTEN (phosphatase tensin homologue) induced by cigarette smoke carcinogens may contribute to the pathogenesis of COPD by causing aberrant inflammatory responses. In 2009, TZORTZAKI and SIAFAKAS [40] proposed that acquired somatic mutations, such as MSI and LOH, may lead to altered lung epithelial barrier cells, which are in turn misinterpreted by the host immune system as "nonself", leading to an aberrant immune response and the clonal expansion of cytotoxic CD8+ cells, ultimately resulting in apoptosis and/or necrosis. Although both our DNA damage hypothesis and the somatic mutation hypothesis require further experimental support, we think that both of these hypotheses suggest a new, previously overlooked role of genetic damage in the pathogenesis of COPD.

In conclusion, the results of the present study strongly suggest that DNA damage underlies the molecular pathogenesis of COPD. The DNA damage hypothesis may help to understand better the pathogenetic mechanism of COPD and to target new drugs, such as drugs to prevent DNA damage and to modulate responses to the DNA damage that leads to the pathobiological processes of COPD.

\section{SUPPORT STATEMENT}

This work was supported by Grant-in-Aid for Scientific Research from the Ministry of Education, Science and Culture, Japan, and by the Ministry of Health, Labour, and Welfare of Japan to investigate intractable diseases.

\section{STATEMENT OF INTEREST}

None declared. 


\section{ACKNOWLEDGEMENTS}

The authors thank M. Shino and Y. Sugimura (First Department of Medicine, Tokyo Women's Medical University, Tokyo, Japan) for their technical assistance.

\section{REFERENCES}

1 MacNee W, Tuder RM. New paradigms in the pathogenesis of chronic obstructive pulmonary disease I. Proc Am Thorac Soc 2009; 6: $527-531$

2 Wistuba II, Lam S, Behrens C, et al. Molecular damage in the bronchial epithelium of current and former smokers. J Natl Cancer Inst 1997; 89: 1366-1373.

3 Mao L, Lee JS, Kurie JM, et al. Clonal genetic alterations in the lungs of current and former smokers. J Natl Cancer Inst 1997; 89: 857-862.

4 Wistuba II, Mao L, Gazdar AF: Smoking molecular damage in bronchial epithelium. Oncogene 2002; 21: 7298-7306.

5 Hecht SS. Progress and challenges in selected areas of tobacco carcinogenesis. Chem Res Toxicol 2008; 21: 160-171.

6 Ciccia A, Elledge SJ. The DNA damage response: making it safe to play with knives. Mol Cell 2010; 40: 179-204.

7 Mah LJ, El-Osta A, Karagiannis TC. $\gamma \mathrm{H} 2 \mathrm{AX}$ : a sensitive molecular marker of DNA damage and repair. Leukemia 2010; 24: 679-686.

8 Bekker-Jensen S, Mailand N. Assembly and function of DNA double-strand break repair foci in mammalian cells. DNA Repair 2010; 9: 1219-1228.

9 Bonner WM, Redon CE, Dickey JS, et al. $\gamma \mathrm{H} 2 \mathrm{AX}$ and cancer. Nat Rev Cancer 2008; 8: 957-967.

10 Kuo LJ, Yang LX. $\gamma$-H2AX - a novel biomarker for DNA doublestrand breaks. In Vivo 2008; 22: 305-309.

11 Rodier F, Coppe JP, Patil CK, et al. Persistent DNA damage signaling triggers senescence-associated inflammatory cytokine secretion. Nat Cell Biol 2009; 11: 973-979.

12 Freund A, Orjalo AV, Desprez PY, et al. Inflammatory networks during cellular senescence: causes and consequences. Trends $\mathrm{Mol}$ Med 2010; 16: 238-246.

13 Schultz LB, Chehab NH, Malikzay A, et al. p53 binding protein 1 (53BP1) is an early participant in the cellular response to DNA double-strand breaks. J Cell Biol 2000; 151: 1381-1390.

14 Bakkenist CJ, Kastan MB. DNA damage activates ATM through intermolecular autophosphorylation and dimer dissociation. Nature 2000; 421; 499-506.

15 Yokohori N, Aoshiba K, Nagai A. Increased levels of cell death and proliferation in alveolar wall cells in patients with pulmonary emphysema. Chest 2004; 125: 626-632.

16 Kasahara Y, Tuder RM, Cool CD, et al. Endothelial cell death and decreased expression of vascular endothelial growth factor and vascular endothelial growth factor receptor 2 in emphysema. Am J Respir Crit Care Med 2001; 163: 737-744.

17 Tsuji T, Aoshiba K, Nagai A. Alveolar cellular senescence in patients with pulmonary emphysema. Am J Respir Crit Care Med 2006; 174: 886-893.

18 Tsuji T, Aoshiba K, Nagai A. Alveolar cell senescence exacerbates pulmonary inflammation in COPD patients. Respiration 2010; 80: 59-70.

19 Zhang HP, Xu YJ, Zhang ZX, et al. Expression of protein kinase C and nuclear factor kappa $B$ in lung tissue of patients with chronic obstructive pulmonary disease. Zhonghua Nei Ke Za Zhi 2004; 43: 756-759.

20 Aoshiba K, Nagai A. Senescence hypothesis for the pathogenetic mechanism of chronic obstructive pulmonary disease. Proc Am Thorac Soc 2009; 6: 596-601.

21 Deslee G, Woods JC, Moore C, et al. Oxidative damage to nucleic acids in severe emphysema. Chest 2009; 135: 965-974.

22 Solier S, Pommer Y. The apoptotic ring. A novel entity with phosphorylated histones $\mathrm{H} 2 \mathrm{AX}$ and $\mathrm{H} 2 \mathrm{~B}$ and activated DNA damage response kinases. Cell Cycle 2009; 8: 1853-1859.

23 Klaunig JE, Xu Y, Isenberg JS, et al. The role of oxidative stress in chemical carcinogenesis. Environ Health Perspect 1998; 1: 289-295.

24 Povirk LF, Han YH, Steighner RJ. Structure of bleomycin-induced DNA double-strand breaks: predominance of blunt ends and single-base 5' extensions. Biochemistry 1989; 28: 5808-5814.

25 Zhang W, Zhu J, Bai J, et al. Comparison of the inhibitory effects of three transcriptional variants of CDKN2A in human lung cancer cell line A549. J Exp Clin Cancer Res 2010; 29: 74.

26 Siafakas NM, Tzortzaki EG, Sourvinos G, et al. Microsatellite DNA stability in COPD. Chest 1999; 116: 47-51.

27 Zervou MI, Tzortzaki EG, Makris D, et al. Differences in microsatellite DNA level between asthma and chronic obstructive pulmonary disease. Eur Respir J 2006; 28: 472-478.

28 Samara KD, Tzortzaki EG, Neofytou E, et al. Somatic DNA alterations in lung epithelial barrier cells in COPD patients. Pulm Pharmacol Ther 2010; 23: 208-214.

29 Zhao H, Albino AP, Jorgensen E, et al. DNA damage response induced by tobacco smoke in normal human bronchial epithelial and A549 pulmonary adenocarcinoma cells assessed by laser scanning cytometry. Cytometry A 2009; 75: 840-847.

30 Zhou F, Onizawa S, Nagai A, et al. Epithelial cell senescence impairs repair process and exacerbates inflammation after airway injury. Respir Res 2011; 12: 78.

31 Rhaman I, Adcock IM. Oxidative stress and redox regulation of lung inflammation in COPD. Eur Respir J 2006; 28: 219-242.

32 Matzinger P. The danger model: a renewed sense of self. Science 2002; 296: 301-305.

33 Kuilman T, Michaloglou C, Vredeveld LC, et al. Oncogeneinduced senescence relayed by an interleukin-dependent inflammatory network. Cell 2008; 133: 1019-1031.

34 Kim V, Rogers TJ, Criner GJ. New concepts in the pathobiology of chronic obstructive pulmonary disease. Proc Am Thorac Soc 2008; 5: 478-485.

35 Walter RE, Wilk JB, Larson MG, et al. Systemic inflammation and COPD: the Framingham Heart Study. Chest 2008; 133: 19-25.

36 Kuhn C 3rd, Homer RJ, Zhu Z, et al. Airway hyperresponsiveness and airway obstruction in transgenic mice. Morphologic correlates in mice overexpressing interleukin (IL)-11 and IL-6 in the lung. Am J Cell Mol Biol 2000; 22: 289-295.

37 Dickey JS, Baird BJ, Redon CE, et al. Intercellular communication of cellular stress monitored by gamma-H2AX induction. Carcinogenesis 2009; 30: 1686-1695.

38 Cosio MG, Saetta M, Agusti A. Immunologic aspects of chronic obstructive pulmonary disease. N Engl J Med 2009; 360: 2445-2454.

39 Anderson GP, Bozinovski S. Acquired somatic mutations in the molecular pathogenesis of COPD. Trends Pharmacol Sci 2003; 24: $71-76$.

40 Tzortzaki EG, Siafakas NM. A hypothesis for the initiation of COPD. Eur Respir J 2009; 34: 310-315. 